19 Revue d'histoire du XIXe siècle

Société d'histoire de la révolution de 1848 et des

révolutions du XIXe siècle

$23 \mid 2001$

Nouvelles approches en histoire économique

\title{
Les économistes français et l'usage des modèles étrangers
}

\section{Lucette Le Van Lemesle}

\section{OpenEdition \\ Journals}

Édition électronique

URL : http://journals.openedition.org/rh19/311

DOI : $10.4000 /$ rh19.311

ISSN : $1777-5329$

Éditeur

La Société de 1848

Édition imprimée

Date de publication : 1 décembre 2001

Pagination : 73-86

ISSN : 1265-1354

Référence électronique

Lucette Le Van Lemesle, « Les économistes français et l'usage des modèles étrangers », Revue d'histoire du XIXe siècle [En ligne], 23 | 2001, mis en ligne le 04 mars 2008, consulté le 03 mai 2019. URL : http://journals.openedition.org/rh19/311 ; DOI : 10.4000/rh19.311

Ce document a été généré automatiquement le 3 mai 2019.

Tous droits réservés 


\title{
Les économistes français et l'usage des modèles étrangers
}

\author{
Lucette Le Van Lemesle
}

1 À l'inverse des penseurs sociaux, les économistes français du XIX ${ }^{\mathrm{e}}$ siècle intéressent trop peu les historiens. Il est vrai qu'ils ont été durablement discrédités, à la fois au XIXe siècle par Marx et au XXe par le plus célèbre des historiens de l'analyse économique, Schumpeter. Pour le premier, leur œuvre se résume à celle d'idéologues vulgaires. Pour le second, ce sont des praticiens efficaces, de bons conseillers s'il s'agit de gérer son patrimoine, mais leur apport en matière d'analyse scientifique est faible. Mais, dans les deux cas, l'exécution symbolique s'est effectuée au nom d'une vérité scientifique acquise une fois pour toutes. On est donc en présence d'une conception téléologique de l'histoire de la pensée selon laquelle les auteurs sont jugés en fonction de leur apport à une vérité scientifique absolue ou du moins considérée comme telle au moment du jugement. Il est évident qu'une étude historique de la pensée économique ne peut procéder ainsi, les auteurs devant être jugés à la fois en fonction de leur contexte, en fonction des médiateurs culturels qui choisissent de diffuser leur pensée et enfin en fonction de leur influence réelle.

2 Une relecture refusant ces anciens a priori les présente sous un aspect très différent : bons théoriciens ou pas, ils observent de très près la réalité de leur temps et proposent de multiples solutions pour la favoriser ou la modifier. Et leur témoignage est également original par leur souci de ne pas dissocier les aspects économiques des aspects sociaux, voire même politiques. Enfin les représentations qu'ils diffusent peuvent susciter des comportements nouveaux, parfois massifs, qui doivent être réintroduits dans l'histoire générale. Notre démarche consiste donc à les utiliser comme source.

3 Parmi les aspects de leurs œuvres nous avons choisi de sélectionner leur attitude face aux pays étrangers le plus souvent considérés comme des modèles économiques : l'Angleterre et les États-Unis. Il ne s'agit évidemment pas de modèle au sens mathématique ou économique actuel. Nous employons modèle au sens d'objet possédant certaines qualités ou caractéristiques propres à en faire le type d'un fonctionnement économico-social, et par conséquent susceptible de devenir un objet d'imitation. 
4 Dans le premier tiers du XIX siècle, trois auteurs (Jean-Baptiste Say, Charles Dupin et Adolphe Blanqui) font la promotion du modèle britannique, mais au travers de la sympathie de ce dernier des inquiétudes commencent à poindre. Aussi dès le début des années 1830 la description du système nord-américain, présenté comme une extension «de la race anglo-saxonne» par Michel Chevalier témoigne, en dépit de l'avance reconnue, de réserves évidentes vis-à-vis de l'industrialisation britannique. Léon Faucher enfin, au début des années 1840, souligne, lui, les limites inacceptables du modèle, mais entrevoit grâce à Chevalier une solution dans les conditions spécifiques du modèle américain ${ }^{1}$.

L'Angleterre, un modèle technique ou un système global ?

5 La fascination à l'égard du pays qui a réalisé le premier la révolution industrielle ne pouvait laisser indifférent toute personne qui s'intéresse à l'économie politique. L'avance économique réalisée avait été confirmée par le rôle de l'Angleterre dans le financement des coalitions contre la France de la Révolution et de l'Empire et par le maintien de sa puissance économique en 1815, en dépit de cet effort de guerre.

6 Parallèlement, même si, pendant les guerres, les transferts de technologie ont été ralentis ${ }^{2}$, ils ont eu lieu quand même. Un nombre relativement élevé de techniciens britanniques se trouvait en France pendant toute la période de la guerre selon J. Harris ${ }^{3}$. Ils ont réduit mais plus lentement qu'en temps de paix l'écart technologique entre l'Angleterre et la France dans les domaines de la métallurgie et du textile ${ }^{4}$.

7 Avec la fin des guerres «il y eut une véritable ruée en Angleterre d'industriels, notamment de maîtres de forges, et d'ingénieurs ${ }^{5}$. On sait que les mécaniciens et les techniciens anglais étaient attirés en France à la fois à cause des difficultés de vie quotidienne chez eux et par les hauts salaires proposés par les patrons français soit pour former leurs propres ouvriers soit pour faire fonctionner les machines britanniques importées en fraude. Sans prendre position ici sur le thème d'une révolution industrielle importée ou spécifique, nous nous proposons de montrer le rôle joué par les économistes les plus reconnus de l'époque face à l'exemple britannique. Dans l'impossibilité de citer tous les auteurs du premier tiers du XIXe siècle qui signalent l'importance de ce fait, nous nous contenterons d'utiliser ceux qui ont eu une véritable notoriété, ont fait l'objet de rééditions et ont donc contribué à la diffusion ou à la mise à distance du modèle anglais ${ }^{6}$. C'est le cas de l'économiste Jean-Baptiste Say, de l'ingénieur et homme politique Charles Dupin et du publiciste Adolphe Blanqui pour la promotion du modèle anglais. Ce modèle anglais reste présent en filigrane à titre de comparaison dans le récit du voyage aux ÉtatsUnis que nous présente Michel Chevaler au début de la monarchie de Juillet. Il est repris en 1841 de façon beaucoup plus détaillée et plus critique par l'économiste et homme politique Léon Faucher. Mais les usages de l'analyse du phénomène britannique aboutissent à des conclusions très différentes et témoignent d'une évolution face à l'industrialisation dans son ensemble.

Pour J.-B. Say (1767-1832), un système économique global où le retard du politique freine l'économique

8 Le premier contact de Jean-Baptiste Say avec la révolution industrielle date de 1784. Il n'a alors que 17 ans. Fils d'un négociant protestant soucieux de donner à ses enfants une formation à la fois théorique et pratique, Jean-Baptiste Say est envoyé en Angleterre avec son frère Horace pour compléter son éducation commerciale et pour apprendre la langue. Le séjour dure presque deux ans. Notre auteur peut observer la rapidité et l'efficacité des mutations économiques engendrées par l'application à l'industrie des découvertes 
techniques dans le domaine du textile et dans les voies de communication. Il n'a jamais eu le temps d'écrire ni de publier ses mémoires comme il en avait l'intention mais, grâce aux manuscrits acquis par la Bibliothèque nationale, nous disposons des notes qu'il a prises à chaud dans ce but. Ses observations portent sur tous les aspects de l'économie anglaise dont il souligne les aspects spécifiques. «On augmente la puissance matérielle par tous les moyens possibles, par l'éducation, par les transports, par des regroupements et par les différents procédés. On favorise l'agriculture d'abord et ensuite les manufactures qui les premières mettent en œuvre les matières du pays ». « $16 \%$ du sol anglais est en friche [...]. Ce qui est cultivé l'est bien " ${ }^{7}$. Dans l'ensemble, la rationalité de la production et de la circulation (entretien des routes minutieusement décrit, règlement sur le chargement des chariots, rôle des canaux) retient son attention. Mais ce manuscrit - pas toujours facile à déchiffrer - est surtout complété par de très nombreux schémas de machines et témoigne de la prise de conscience par le jeune Say de l'importance des aspects techniques de la révolution industrielle.

9 L'adolescent n'en garde pas moins sa part d'esprit critique face aux méfaits de la fiscalité britannique. Il dénonce en particulier l'absurdité de l'impôt sur les portes et fenêtres qui amène son propriétaire, pour diminuer ses impôts, à obscurcir en partie la chambre qu'il loue aux deux jeunes Français. Plus globalement, s'ils acceptaient d'alléger leur fiscalité, « les Anglais profiteraient considérablement de cette grande supériorité qu'ils ont dans les procédés de fabrication, supériorité qui dans l'état présent des choses est balancée et neutralisée par l'énormité de leurs dépenses publiques " 8 .

Une trentaine d'années plus tard, en 1814, on retrouve la même démarche, mais plus systématisée. Cette fois, c'est un système économique global dont il s'agit de montrer les forces et les faiblesses. Après la chute de Napoléon, l'économiste reconnu qu'est devenu J.-B. Say ${ }^{9}$ est chargé par le gouvernement provisoire de faire un voyage d'étude en Angleterre afin de rendre compte de sa situation économique, après les guerres de la Révolution et de l'Empire. De retour, il publie en 1815, une brochure «De l'Angleterre et des Anglais » qui remporte un succès suffisant pour être l'objet de deux nouvelles éditions l'année suivante, en $1816^{10}$. Grâce à la paix retrouvée, nous explique-t-il, « on a pu aller chercher de l'autre côté de la Manche l'explication de plusieurs phénomènes dont on ne connaissait que les résultats, et mesurer le levier qui, plus d'une fois a soulevé l'Europe " ${ }^{11}$. La cause n'est pas la quantité d'or détenue par l'Angleterre qui pourrait expliquer la puissance maintenue du monde britannique, mais «c'est par sa richesse et son crédit qu'elle a pu agir ; et comme ces armes puissantes sont le résultat de toute son économie, c'est son système économique qui est son trait saillant, et qui mérite de fixer notre attention " ${ }^{12}$. Ce système se manifeste par des profits commerciaux « qui se sont accrus à un point surprenant », par l'accroissement remarquable de la population des villes, maritimes ou non, par de nouvelles constructions, par l'enrichissement des fermiers qui doivent nourrir les villes. "L'introduction des machines dans les arts [...] a rendu la production des richesses plus économique », les machines à vapeurs se sont généralisées, on utilise le « charbon de terre ».

11 Say constate même que la "dépréciation" (dévalorisation) de la monnaie anglaise augmente les commandes étrangères et stimule l'activité du pays. En effet cette dépréciation permet aux spéculateurs étrangers de s'enrichir en payant des marchandises anglaises en monnaie anglaise dévaluée sur le continent (la livre y vaut dix-huit francs au lieu de vingt-quatre) et de gagner au moins $25 \%$ sur le seul taux de change. 
gabal particulièrement efficace en matière de production, mais il ne peut dans l'immédiat être un modèle économique global à imiter. Pour Say, le progrès économique, l'augmentation des richesses, n'ont de sens qu'au service des besoins sociaux, du «bien public ». Pour l'ancien girondin, pour l'Idéologue qu'est Say, seul le recul de la misère rend possible la mise en œuvre de la liberté politique. La fonction de l'économie politique est d'être au service de l'idéal de la Révolution en lui permettant de s'incarner dans les faits. À l'aube, en France, de la première révolution industrielle, le modèle anglais bien compris pour sa puissance technique est mis à distance au plan de ses effets néfastes sur le niveau de vie des populations. Séquelles de colonialisme, protectionnisme source de vie chère, et engagement trop actif dans les guerres depuis 1793 ont grevé les finances publiques et appauvri lourdement les habitants de ce pays riche. Les limites du modèle sont le résultat d'une mauvaise politique: elles sont purement conjoncturelles. En dernière analyse les qualités et les limites de l'exemple anglais illustrent le bien fondé d'un véritable libéralisme qui reste à mettre en œuvre. À ce moment-là seulement les forces productives libérées seront mises réellement au service des besoins des populations. Cette analyse du cas anglais sert donc à réaffirmer les vérités scientifiques fondamentales à mettre au service de l'implantation en France de la première révolution industrielle, en lui épargnant les erreurs commises en Angleterre.

Un programme commun pour réunir les Français ? profiter de l'expérience anglaise pour reconstruire la France ou les voyages de Charles Dupin

Beaucoup plus simple, mais aussi techniquement plus rigoureux est le discours proposé par l'ingénieur Charles Dupin (1784-1873). Ses «Voyages dans la Grande-Bretagne entrepris relativement aux services publics de la guerre, de la marine et des Ponts et Chaussées, en 1816, $1817,1818,1819$ et $1820 "{ }^{13}$ paraissent en 1821 et 1824 et ont assez de succès pour être réédités en 1825-1826. Lui aussi est fasciné par l'avance technique de l'Angleterre.

Si la période est presque la même que pour le voyage anglais de Say, le contexte personnel de l'auteur est très différent. Ce polytechnicien, membre du corps des ingénieurs de la marine, a fait carrière au temps de l'Empire ${ }^{14}$. Mais, tout en menant une carrière militaire, il manifeste des préoccupations à la fois pédagogiques et scientifiques. Dans ce contexte post-révolutionnaire, il est persuadé que l'éducation est la première source de tous les progrès. Dans ce but, il avait tenté de faire vivre à Corfou, où il était en garnison, une Académie ionienne, sorte d'institution d'éducation sur le modèle de l'Institut d'Égypte. Les succès avaient été très limités. Au plan scientifique, il est très tôt remarqué 
par Lazare Carnot et par Monge. Il publie en 1813 un ouvrage de recherche " Développement de la géométrie pour faire suite à la géométrie descriptive et à la géométrie analytique de M. Monge ». En 1815, tout en n'étant pas un opposant systématique à la Restauration, il refuse de renier ces amitiés scientifiques, ce qui le rend suspect au nouveau gouvernement. Aussi, lorsqu'il sollicite le droit de se rendre en Grande-Bretagne pour y étudier les réseaux de communication, les fortifications militaires et l'économie industrielle, doit-il attendre dix mois l'autorisation.

Durant quatre années de 1815 à 1819, il multiplie les voyages scientifiques en Angleterre et en publie les résultats dans des rapports à l'Académie des sciences, les Mémoires sur la marine (1818) et dans les six volumes des Voyages dans la Grande Bretagne (1820-1824). Il visite les ports, les principales villes industrielles d'Angleterre et d'Écosse et s'attarde dans les trois capitales: Londres, Édimbourg et Dublin. Il ne s'agit plus d'analyser un système économique dans tous ses aspects, mais de mettre à la portée des Français des descriptions techniques très précises, souvent accompagnées de plans cotés des ponts, des routes, des docks, des ouvrages d'art de toutes sortes souvent complétés par des planches de dessin industriel. « Aux yeux du sage, la puissance des nations est un fait qu'il étudie comme un naturaliste étudie un phénomène, comme le géomètre étudie les vérités mathématiques : pour en connaître les principes et pour en découvrir les conséquences » ${ }^{15}$. Seules les introductions et les conclusions proposent une interprétation des causes de la puissance ainsi décrite avec un soin méticuleux. "Quels moyens, quels travaux ont produit cette force elle-même? Des travaux pareils, des moyens analogues pourraient-ils élever d'autres peuples au même degré de puissance? Voilà ce qu'il nous importe de savoir, comme Français, pour l'avantage de la France ${ }^{16}$.

Il souligne les spécificités historiques et sociales de la Grande Bretagne, le développement lié au grand commerce, la participation de tous les groupes sociaux (même les grands propriétaires) à l'expansion, «pas de jalousie des classes laborieuses » affirme-t-il. Mais il souligne qu'il y a 50 ans l'Angleterre était en retard: "Nous pouvons reprendre notre rang en profitant de son expérience comme elle a su profiter de la nôtre [...] Nous invitons nos concitoyens à suivre la route où depuis un demi-siècle, l'Angleterre a marché pour son bonheur et sa fortune", ce qui permettrait en outre de "ramener la concorde publique, la paix intérieure par le bien-être général ». Il s'agit donc de ramener la paix sociale en France en lui proposant un programme commun de développement.

On pourrait penser qu'à surfer ainsi sur la vague anglophile Dupin fait preuve d'un simple opportunisme utilitaire. En fait sauf pendant le ministère Decazes, sous la Restauration, Dupin reste dans une relative opposition. Il a obtenu comme Say, une chaire au Conservatoire royal des Arts et Métiers, ce qui lui permet de s'adresser à un public plus large que celui des lecteurs habituels. Mais dès 1820 son cours est surveillé par la police et son livre est retiré des bibliothèques de l'armée... pour apologie de l'ennemi. Comme Say, il ressent la monarchie de Juillet comme l'incarnation de ses espoirs et c'est là qu'il construit l'essentiel de sa carrière politique. Mais à la différence des libéraux organisés autour de Say, la puissance britannique lui paraît telle qu'il reste partisan du protectionnisme pour la France. Dans les deux cas, chez Say comme chez Dupin, le modèle global, grâce à sa puissance technique, ne se discute pas.

De l'enthousiasme à l'inquiétude : les premières ombres au tableau

20 Curieusement, c'est chez un observateur enthousiaste, Adolphe Blanqui (1798-1854), quasi amoureux du libéralisme à l'anglaise, que des inquiétudes sérieuses commencent à poindre, dès son voyage de 1823, qui se confirment par la suite. 
$21 \mathrm{Au}$ premier abord, l'Angleterre apparaît au jeune voyageur comme un modèle de libéralisme dans tous ses aspects. En 1823, A. Blanqui, âgé de 25 ans, fait partie du cercle d'amis proches de J.-B. Say " son ami, son maître ", dont il partage les idées et le carnet d'adresses. Il entretient avec lui une correspondance suivie et lui succède dans divers lieux d'enseignement, à l'Athénée Royal et au Conservatoire des arts et métiers. À l'automne 1823, Il fait un voyage de trois mois et publie, en 1824, Le Voyage d'un jeune Français en Angleterre et en Écosse pendant l'automne $1823{ }^{17}$.

Même si les deux auteurs précédents, Say et Dupin lui ont servi à préparer son voyage, même s'il cite le rôle du chimiste Nicolas Clément-Désormes ${ }^{18}$, le genre du livre est différent. Sur le modèle du voyage romantique, il a choisi le récit du voyage économique, associant l'observation informée, la réaction subjective qu'elle provoque et la leçon idéologique qu'on peut en tirer. "Cette relation n'est que l'expression sincère de ce que j'ai éprouvé, et quelque fois j'ai eu des moments d'exaltation ou des accès de tristesse. On ne voyage pas sans un peu d'enthousiasme chez un peuple aussi grand, sans un peu d'amertume chez d'aussi anciens ennemis ${ }^{19}$.

Dès le départ, à la sortie du Havre, en quittant la France des Ultras, l'auteur ressent un climat de liberté, de libération de la surveillance policière. « Nous sortons du port comme d'une prison ». Le premier contact avec l'Angleterre est celui d'un pays libre, propre et prospère où « les campagnes présentent partout l'aspect d'un grand jardin entrecoupé de haies vives, parsemées de beaux arbres ${ }^{20}$. Les gens du peuple sont décemment vêtus, toutes les femmes même celles de la dernière classe ont des chapeaux... » ${ }^{21}$.

Les discussions et les débats sont libres, la police est peu nombreuse sans que le désordre règne. « Mais ne pourrait-on pas supposer avec raison encore que plus un peuple travaille et que plus il s'éclaire, moins il produit de ces misérables poussés à l'infamie par les besoins ou par l'absence de toute éducation " ${ }^{22}$. Les intérêts individuels "disparaissent devant la seule utilité publique ». Bref, on est au paradis du libéralisme politique.

Mais si l'auteur refuse le tourisme traditionnel, la visite des bijoux de la couronne par exemple (ce vain appareil a perdu de son influence), en revanche dans ce long tour de l'Angleterre et de l'Écosse, il souligne la prospérité des villes, leur éclairage au gaz, leur salubrité (il décrit l'enlèvement des ordures), les belles places, l'urbanisme. Il s'attache au mouvement économique et à ses lieux symboliques, la beauté de la Bourse de Londres par exemple : «C'est un des plus beaux édifices de la cité » ${ }^{23}$. Londres est décrite au travers de ses quatre secteurs correspondant à quatre fonctions économiques: la rive droite ( Southwark) où les manufactures assurent la production, la Cité au centre qui enregistre les transactions dans ses comptoirs, les beaux quartiers de l'Ouest et leur fonction de consommation, l'Est enfin, lieu du commerce avec ses bassins, ses entrepôts, ses ports et ses vaisseaux. Les docks de Londres sont l'objet d'un morceau de bravoure sur la puissance britannique que l'auteur rêve de " voir un jour réalisée sur la terre de France ».

Les premiers contacts avec le monde de l'industrialisation n'atténuent pas immédiatement son enthousiasme. Le travail des enfants dans les mines de la région de Glasgow est signalé mais réduit à une description pittoresque. "C'est un spectacle imposant et bizarre que celui d'une galerie de mine. Ces cyclopes vont et viennent, éclairés par la mèche qui brûle sur leur front, ces enfants tout noirs, tout nus et bien portant, ces hommes ensevelis pendant une partie de leur vie inspirent des émotions qu'on ne retrouve que dans les catacombes $"{ }^{24}$. Le ton change avec la description des villes embrumées par les fumées « Bristol, ville de fumée de briques et de boue [...] riche 
cloaque plus encore que Rouen à laquelle elle ressemble [...] un monceau de briques dominé par des nuages de fumées au milieu desquelles s'élèvent quelques flèches de clocher et des cônes tronqués d'une forme et de couleur dégoûtants. Ce sont les fourneaux où l'on fabrique les glaces ${ }^{25}$.

Mais ce sont les descriptions du paysage minier et métallurgique dans la région de Birmingham qui lui font mesurer le poids social de l'activité industrielle. Après avoir montré la laideur romantique d'un sol « cicatrisé par les mines », le rôle de la poussière qui teinte gens et maisons, ces « cyclopes » qui ont fourni de l'armement à toute l'Europe pendant vingt ans de guerre, l'auteur s'interroge sur la vie des ouvriers de la métallurgie. "Les habitants semblent ignorer qu'il est des douceurs dans la vie, qu'un petit jardin, une pelouse et des fruits, une maison propre et commode font le bonheur de leurs compatriotes à dix lieues de distance [...]. Pour la première fois dans cette plaine, j'ai commencé à comprendre l'industrie anglaise ${ }^{26}$.

Même si le livre se conclut sur une note optimiste - « Nous venons de visiter le séjour de l'industrie et de l'opulence »-, cette compréhension du coût social de l'industrialisation s'accentue dans l'évolution de Blanqui et s'exprimera de plus en plus nettement dans son enseignement au Conservatoire des arts et métiers où un public ouvrier venait assister à son cours pour entendre parler de la vie des fabriques ${ }^{27}$.

Dans ce livre enthousiaste où consciemment l'Angleterre est présentée comme modèle de libéralisme politique et de modernité économique une ombre sérieuse vient entacher le portrait du premier pays industrialisé. Il n'est pas un modèle de libéralisme social au sens où l'entendent les libéraux qui dans la mouvance de J.-B. Say et Adolphe Blanqui veulent mettre la libération des blocages issus de l'Ancien Régime au service du progrès de la société tout entière.

La prise de conscience du paupérisme et le modèle américain

30 Au début de la monarchie de Juillet, une prise de conscience des limites sociales du modèle anglais s'impose. Mais les allusions y sont peu nombreuses face à l'importance de son avance, en particulier en matière de chemin de fer, dans l'œuvre de Michel Chevalier. C'est seulement Léon Faucher qui au début des années 1840 ose faire une description précise et implacable de l'ampleur du paupérisme en Angleterre et, quelques années après, systématise, plus que Chevalier, l'idée d'un modèle américain.

Les États-Unis d'Amérique et Michel Chevalier (1806-1879) ${ }^{28}$

31 En 1832, Michel Chevalier, à 26 ans, est envoyé par Thiers aux États-Unis. Sa mission consiste à y étudier la place des travaux publics. Polytechnicien et ingénieur des mines, il vient à peine de sortir de quelques mois de prison pour participation à une association illicite. Rallié au saint-simonisme du " père » Enfantin, il en avait partagé les aspects les plus extrêmes : vie dans une communauté à Ménilmontant, cantiques, manifestations de rue en costume quasi sacerdotal, communauté des femmes, etc. Son retour au Ministère des Travaux Publics et cette mission très officielle sont pour lui l'occasion de démontrer qu'il est rentré dans le rang. Mais il est resté très marqué par les aspects économiques de la pensée saint-simonienne. Ce voyage donne lieu à des lettres publiées dans le Journal des Débats puis regroupées dans un ouvrage en deux tomes, les Lettres sur l'Amérique du Nord ${ }^{29}$.

Il insiste d'abord sur l'intérêt du voyage économique. Il ne s'agit pas d'imiter ces touristes légers qui ont perçu l'industrie "à la façon d'un décor d'opéra ». Il ne suffit pas de constater et d'admirer les innombrables vaisseaux d'un pays mais de se demander comment et pourquoi il a accédé à l'opulence. Une entreprise de brasserie «à la marche 
parfaite, [...] qui fait travailler 300 personnes » a plus d'importance que les châteaux romantiques, ces « ruines féodales». On est dans une perspective historique de marche vers la modernité. Dans un vaste panorama du progrès ou de la décadence des civilisations, l'auteur souligne les progrès de civilisation accomplis par les Anglais et par la « race anglo-saxonne » en général au détriment des races méditerranéennes. Il est urgent pour la France d'assimiler ces progrès et éventuellement de servir de médiateur pour les transmettre aux races méditerranéennes. Les Américains sont les « rejetons » et les continuateurs des Anglais dans le Nouveau Monde.

Les deux premières lettres s'intitulent "le chemin de fer de Paris à Londres " et «Liverpool et son chemin de fer ». Elles témoignent encore d'une fervente anglophilie. Les Anglais sont des commerçants et des entrepreneurs modèles. Leurs villes sont modernes, cossues, éclairées au gaz. Les trains rapprochent à la fois les lieux et les classes sociales. Le modèle anglais sert ici à promouvoir en France la nécessité pour le gouvernement de s'intéresser aux chemins de fer.

Aux États-Unis, les communications, le développement industriel, la modernité des campagnes et la prospérité des villes, tout a été rendu possible grâce au système bancaire. Il a servi de "levier pour installer chez eux au profit de toutes les classes sociales l'agriculture et l'industrie de l'Europe et pour couvrir leur sol de routes, canaux, usines et temples, et en un mot tout ce qui constitue la civilisation ${ }^{30}$. Les banques sont hiérarchisées, organisées en système et la banque centrale contrôle les banques locales. Le progrès technique, le rôle des machines à vapeur démontrent la puissance humaine. Les «machines locomotives" par exemple sont évoquées avec lyrisme. "Cela marche, cela court comme un cheval ventre à terre. Bien plus, cela respire : la vapeur qui sort périodiquement des cylindres et qui se condense en fumée blanche ressemble véritablement au souffle précipité d'un cheval de course »; ou, plus loin, « on est tenté de croire à un dragon ailé vomissant feu et flamme ${ }^{31}$.

Le rôle dominant du travail, le mépris à l'égard de l'oisiveté, les aspects particuliers de la religion, de l'esprit puritain ont favorisé une industrialisation qui semble échapper à la malédiction du paupérisme. Il concède à ce propos les limites du modèle anglais. « Temporairement le système manufacturier a des inconvénients terribles. C'est la plaie de l'Angleterre ${ }^{32}$. C'est pourquoi il expose avec tant de précision l'expérience de Lowell. "L'établissement d'un système manufacturier sur une terre nouvelle est un fait digne de la plus haute attention [...] pour savoir jusqu'à quel point la création d'une manufacture avait suscité ici à l'égard de la sécurité des riches et de l'ordre public les mêmes dangers qu'en Europe ${ }^{33}$. Les ouvrières de la fabrique de coton de Lowell sont des filles de fermiers de la région qui viennent travailler pour se constituer une dot. Or elles sont beaucoup mieux payées qu'en Europe, elles respirent l'aisance et le calme à l'abri d'une vie dans un internat confortable qui les protège sans les surexploiter. Chevalier explique l'attitude patronale par le rôle de la mentalité puritaine, et de l'éducation protestante qui trace autour de chaque individu un cercle de respect mutuel. Mais il signale aussi que la pénurie de main d'œuvre n'est pas sans rapport avec le niveau des salaires. Il en tire la conclusion que c'est un phénomène particulier au nouveau monde.

En général, d'ailleurs, la description des États-Unis sert à les faire connaître mais non à les imiter mécaniquement. C'est le cas dans le domaine politique. La démocratie américaine n'est pas plus exportable telle quelle en France que le système bancaire ou l'aisance des classes moyennes. L'exemple américain sert à réfléchir, mais doit être adapté aux caractères de chaque nation. Qu'il s'agisse de mettre en place le système 
bancaire par exemple si nécessaire en France, on ne peut pas se fier à la seule initiative individuelle, l'État devra intervenir. Le suffrage universel lui non plus n'est pas utile immédiatement dans un pays catholique comme la France. La monarchie sera moins implacable vis-à-vis des plus pauvres que ne le serait la seule bourgeoisie "peu généreuse » au pouvoir. Dans l'ensemble la priorité doit être mise sur un développement économique contrôlé et organisé si on veut " améliorer le sort du plus grand nombre ». Il y a ici en fait une remise en cause du libéralisme pur, l'excès de libertés ne convenant pas à un pays catholique monarchique et centralisé comme la France, peu habitué à l'autodiscipline. «Les États-Unis peuvent se passer d'un César, nous sommes nous obligés de le garder ${ }^{34}$. Mais la France peut s'inspirer du progrès du machinisme et développer la culture technique qui a si bien réussi aux « rejetons » des Anglais.

En somme les États-Unis sont une source de réflexion utile, mais jamais un modèle à copier tel quel. La spécificité de chaque pays doit être respectée, aucun modèle absolu n'étant généralisable.

L'Angleterre, exemple des méfaits de son industrialisation

Tout autre est l'attitude de l'économiste et homme politique Léon Faucher (1803-1854) au début des années 1840 . Le contexte social et politique est différent. On se préoccupe à la Chambre, sous la pression des pétitions, du sort des ouvriers des manufactures. C'est le moment où le Ministère du Commerce ordonne une enquête et où l'Académie des sciences morales et politiques charge Villermé de lui faire un rapport sur l'état de la classe ouvrière dans les manufactures. La loi du 22 mars 1841 sur le travail des enfants dans les manufactures vient d'être votée. Dans ce contexte, le journaliste libéral Léon Faucher, proche de l'économiste Wolovski son beau frère, trouve intéressant d'aller étudier la situation en Angleterre. Il est lié aux Whigs. Il publie le résultat de son voyage en partie dans la Revue des Deux Mondes, puis sous forme d'ouvrage en $1845^{35}$.

40 L'introduction insiste lourdement sur la singularité britannique, sur leur mépris de l'étranger et sur leur tendance à cultiver l'exclusion. La France a tort d'aller chercher à l'étranger des modèles sous prétexte que l'avance anglaise l'a amené sur le devant de la scène. Mais ce pays original peut servir de « champ d'expérience assez vaste pour étudier les problèmes du présent qui pèsent sur les sociétés modernes", en particulier les problèmes sociaux.

41 L'auteur ne sous-évalue ni la puissance de l'industrie britannique, ni sa force et sa richesse économique, ni l'ampleur de son urbanisation. Mais ses problèmes sociaux ont cru avec la même ampleur. Et la description des différentes formes du paupérisme dans les principales métropoles industrielles est précise, nourrie de statistiques, et sonne comme une dénonciation. De Birmingham à Glasgow, de Manchester à Leeds, tous les centres manufacturiers ont leurs quartiers misérables et délinquants. Londres aussi, en particulier l'East End concentre les quartiers les plus dangereux comme les plus misérables. La description détaille les caractères particuliers de chaque type d'immigrants. Dans le district de Bethnal-Green, la misère a réduit « la race à la taille de Liliputiens ». Y existe deux fois par semaine un marché aux enfants où les parents viennent les louer comme rattacheurs. Le sous-prolétariat irlandais squatte les maisons insalubres et promises à la démolition.

42 À White Chapel c'est le monde des brocanteurs et des marchands; les ordures encombrent la rue, les taudis sont surpeuplés mais rapportent $20 \%$ aux propriétaires, le tout sur fond de prostitution, de vols et de meurtres si bien que la police ne peut pénétrer dans certains quartiers. La mortalité y est précoce et les épidémies nombreuses. Cette 
misère existe même à proximité des "beaux quartiers " puisque des jeunes femmes dorment dehors dans Hyde Park. Les solutions de charité ne suffisent pas et la prison sert souvent de solution. Les crises augmentent le nombre des assistés, dépassent tous les moyens des services sociaux et déplacent la délinquance vers les campagnes. Quand elles échappent à cette délinquance, les « classes inférieures » se partagent entre la révolte, la fascination pour le Chartisme, ou espèrent en les Trade-Unions.

Mais après ce réquisitoire, l'auteur concède qu'il peut s'agir d'une période de transition ${ }^{36}$, d'une sorte d'enfantement d'une société nouvelle puisque l'Angleterre n'a pas le monopole de ces malheurs. Il semble que «ces nids à fièvre " ${ }^{37}$ accompagnent comme période de transition l'industrialisation. D'une part, en Angleterre, une mutation est en cours avec la campagne pour le libre échange, menée par la classe moyenne ascendante sous la direction des « économistes » (synonymes de libéraux). Elle lutte pour reconquérir l'influence politique, et défend l'idée que le libre échange, baissant le prix du blé et du pain, diminuerait la misère. La ligue contre les Cornlaws est une organisation puissante qui tient l'aristocratie foncière en respect. D'autre part, pour la France, dans l'édition de 1845 , l'auteur propose comme remède, à côté de quelques institutions de prévoyance, de disperser les manufactures pour "s'attacher à diminuer les contacts des ouvriers entre eux ${ }^{38}$. Seule la manufacture rurale peut devenir une sorte de communauté, "une association étroite et permanente entre le maître et l'ouvrier ${ }^{39}$.

Plus tard seulement, dans un mémoire pour l'Académie des sciences morales et politiques, publié dans l'édition de 1856, Léon Faucher revient sur l'idée d'un modèle américain en reprenant et en détaillant l'histoire du développement de Lowell. Il oppose le Vieux Monde qui, en 60 ans, a produit Manchester, sa puissance et son cortège « hideux » de malheurs et le Nouveau Monde qui « dans une société sans legs du passé, a enfanté Lowell en 15 années, c'est-à-dire l'activité sans désordre, l'aisance des travailleurs et la régularité des mœurs à côté de la richesse ${ }^{40}$.

Il souligne qu'il s'agit d'une industrie utilisant de la main d'œuvre rurale, occupée pour un temps limité dans l'industrie. Il rêve alors d'un « service manufacturier temporaire ", la majorité du temps restant occupée à l'agri-culture. Ainsi « les populations n'iront plus en dégénérant, et la faiblesse du corps ne coïncidera plus avec le progrès des Lumières, ni l'extrême pauvreté des uns avec la richesse extravagante des autres ${ }^{41}$.

En cette période de romantisme, les économistes ont refusé le goût des ruines et de la nature pour maintenir le genre du voyage économique. Affichant un souci d'enquête scientifique, ils n'ont pas résisté à la quête de modèles. Mais en quarante ans, la révolution industrielle en France s'est implantée avec ses formes spécifiques. Quand le retard français est encore très évident, ils ont cherché à diffuser les principaux aspects d'une puissance technique indiscutable, même s'il fallait mettre en garde contre les mauvaises politiques qui en freinaient l'efficacité. Ils ont contribué à la prise de conscience de la nécessité des transferts de technologie. Mais peu à peu s'ébauchent une réserve puis un refus de l'Angleterre comme modèle, tant sont lourdes les conséquences sociales de ce premier exemple d'industrialisation. Au milieu du siècle, certains économistes se demandent si le monde américain, cette extension de la "race anglosaxonne » peut éventuellement, sous certaines conditions, récupérer la place laissée vacante. Mais les conditions économiques de la France ne sont plus les mêmes. La 
première révolution industrielle est largement avancée et depuis 1840 , et malgré les crises, la croissance s'est accélérée. Et on peut se demander si, au travers de cet étonnant retour sur l'exemple américain, ce n'est pas paradoxalement le modèle français avec ses petites entreprises et ses industries rurales qui est en réalité recommandé.

\section{NOTES}

1.. Pour les pays germaniques, avant 1871 , le modèle n'est plus économique, mais disciplinaire. Ces pays représentent un exemple à imiter de mise en œuvre des sciences camérales ou sciences du gouvernement. Mais cette notion dépasse le droit public en associant les formes nouvelles du droit administratif, constitutionnel avec la discipline de l'économie politique. Il s'agit alors de fonder une discipline nouvelle, les sciences politiques, en démontrant l'utilité de la faire enseigner soit par les facultés de droit, comme dans les pays germaniques, soit dans des facultés spéciales, ou encore dans une école privée ou publique d'administration. On sait que la fondation de l'École libre des sciences politiques, dans le contexte de 1871, a apporté sa solution en dépit des efforts des républicains pour fonder une école publique d'administration. À la fin du XIX ${ }^{\circ}$ la législation sociale de l'Angleterre des Trade-Unions et de l'Allemagne bismarkienne retiennent à nouveau l'attention des économistes. La perception de la force économique de l'Allemagne impériale semble avoir été relativement tardive. Mais l'étude de la façon dont fut utilisé le modèle allemand dépasserait les dimensions d'un article. Voir Claude DIGEON, La crise allemande de la pensée française (1870-1914), Paris, Presses universitaires de France, 1959.

2.. Maurice LÉVY-LEBOYER [dir.], Histoire de la France industrielle, Paris, Éditions Larousse, 1996, pp. 65-67.

3.. John HARRIS, Essays in industry and technology in the eighteenth Century/England and France, Aldershot, Scholar Press, 1992.

4.. Serge CHASSAGNE, Le coton et ses patrons en France, Paris, Éditions de l'École des hautes études en sciences sociales, 1991.

5.. Maurice LÉVY-LEBOYER [dir.], Histoire de la France industrielle, ouv. cité, p. 62.

6.. Cet article ne prétend en rien traiter ce vaste sujet de manière exhaustive ; son but est seulement de lancer des pistes de recherche.

7.. Jean-Baptiste SAY, Notes sur l'Angleterre (août-septembre 1784) ; Bibl. nat. (bibliothèque nationale), 115-117, Ms D.98.21.

8. MF 7268, Notes sur les manufactures anglaises, pp. 234-35.

9.. Jean-Baptiste SAY, Traité d'économie politique, ou simple exposition de la manière dont se forment, se distribuent et se composent les richesses, Paris, Crapelet, $1803 ; 2^{\mathrm{e}}$ édition modifiée : Paris, chez Déterville, 1814.

10.. Jean-Baptiste SAY, De l'Angleterre et des Anglais, Paris, Bertrand, 1815.

11.. Jean-Baptiste SAY, De l'Angleterre et des Anglais, édition de Philippe Steiner, dans JeanBaptiste SAY, Cours d'économie politique et autres essais, Paris, Éditions Flammarion, 1996, pp. 47-80, citation p. 49.

12.. Ibidem. 
13.. Charles DUPIN (Baron), Voyages dans la Grande-Bretagne entrepris relativement aux services publics de la guerre, de la marine et des ponts et chaussées, en 1816, 1817, 1818, 1819 et 1820, Paris, Bachelier,1821-1824, 5 volumes.

14.. Robert FOX, «Charles Dupin », dans Les professeurs du Conservatoire national des Arts et Métiers, Paris, Institut nationale de la recherche pédagogique, 1994, pp. 469-478.

15... Charles DUPIN (Baron), Voyages dans la Grande-Bretagne, ouv. cité, tome 5, p. XIII.

16.. Idem, p. XIV.

17.. Adolphe BLANQUI, Voyage d'un jeune Français en Angleterre et en Écosse pendant l'automne de 1823, Paris, Dondey-Pré père et fils, 1824.

18.. « Personne ne contestera que nos manufactures n'aient fait d'énormes progrès depuis que les fabricants ont mieux connu les grands procédés de l'industrie anglaise. M.

Clément-Desormes, professeur de Chimie au Conservatoire des arts et métiers est un des savants auxquels les fabriques françaises ont le plus d'obligations sous ce rapport :

chacun de ses voyages est une conquête et il voyage souvent »; Adolphe BLANQUI, Voyage d'un jeune Français..., ouv. cité, p. VIII.

19.. Idem, p. IV.

20.. Idem, p. IX.

21.. Idem, p. 12.

22.. Idem, p. 326.

23.. Idem, p. 313.

24.. Idem, p. 197.

25.. Idem, p. 53.

26.. Idem, p. 84.

27.. Francis DÉMIER Adolphe Blanqui, Mémoire de maîtrise sous la direction de Philippe Vigier, l'Université Paris 10-Nanterre. Voir aussi Francis DEMIER, « Blanqui », dans Les professeurs du Conservatoire national des Arts et Métiers, ouv. cité.

28. René RÉMOND, Les États-Unis devant l'opinion française (1815-1852), Cahiers de la Fondation nationale des sciences politiques $n^{\circ} 116-117$, Paris, Librairie Armand Colin, 1962, ne consacre que quelques paragraphes à cet ouvrage économique. Pour les mêmes raisons, nous laissons délibérément à l'écart l'œuvre de Tocqueville dont les préoccupations sont plus politiques qu'économiques.

29.. Michel CHEVALIER, Lettres sur l'Amérique du Nord, Paris, Gosselin, 1836 et Bruxelles, Hauman et $\mathrm{C}^{\circ}, 1837,2$ volumes. Les deux éditions sont rigoureusement semblables.

30. Michel CHEVALIER, Lettres sur l'Amérique du Nord, ouv. cité, tome 1, p. 36.

31.. Ibidem, p. 223.

32. Ibidem, p. 224.

33. Ibidem, p. 226.

34.. Idem, tome 2, p. 212.

35.. Léon FAUCHER, Études sur l'Angleterre, Paris, Guillaumin, 1845. Une deuxième édition est publiée par sa femme, après sa mort en 1856, complétée par des études qu'il a présentées à l'Académie des sciences morales et politiques.

36.. Léon FAUCHER, Études sur l'Angleterre, ouv. cité, p. 312.

37. Ibidem, p. 294.

38. Ibidem, p. 314.

39. Ibidem, p. 345.

40. Léon FAUCHER, Études sur l'Angleterre, Paris, Guillaumin, 1856, 2 volumes, p. 474.

41. Ibidem, p. 491. 


\section{RÉSUMÉS}

Vers 1815, quand la paix revient, les économistes français (Say et Dupin) participent au mouvement d'introduction des techniques en faisant de la Grande-Bretagne un modèle à la fois technique et un système global. A partir de 1824 et au début des années 1830, si le modèle britannique continue d'être admiré, Adolphe Blanqui et surtout Michel Chevalier ont conscience de ses limites sociales. Le modèle américain, variante du modèle anglais montre qu'on peut faire des adaptations nationales originales. Au milieu des années 1840, l'ampleur du paupérisme qui accompagne l'industrialisation anglaise est telle que Léon Faucher démontre sans peine que l'Angleterre n'est pas un modèle. Mais la situation américaine montre que la recherche de modèle n'est pas dénuée d'ambiguité et suit de près l'évolution française.

In 1815 and after, when peace came back, French economists (Say and Dupin) gave an impulse to introduce new English techniques in France, writing accounts of their trips to Britain. Afterwards, from 1824 onwards, and in the early 1830s, the English model was still admired, but Adolphe Blanqui and, above all, Michel Chevalier were aware of its social limits. The American model, which was a variant of the English one, showed that original national adaptations could be made. In the mid-1840s, the extent of pauperism resulting from industrialisation in Britain was such that Léon Faucher easily demonstrated that it was not a model. But the American situation showed that the research of a model was not devoid of ambiguity.

\section{AUTEUR}

\section{LUCETTE LE VAN LEMESLE}

Professeur à l'Université de Marne la Vallée 